\title{
Multicomponent $\mathrm{He}$ I $10830 \AA$ profiles in an active filament
}

\author{
C. Sasso ${ }^{1,2}$, A. $\operatorname{Lagg}^{1}$, and S. K. Solanki ${ }^{1,3}$ \\ 1 Max-Planck-Institut für Sonnensystemforschung, Max-Planck-Str. 2, Katlenburg-Lindau, Germany \\ e-mail: csasso@oacn.inaf.it \\ 2 INAF - Osservatorio Astronomico di Capodimonte, Salita Moiariello 16, 80131 Napoli, Italy \\ 3 School of Space Research, Kyung Hee University, Yongin, 446-701 Gyeonggi, Korea
}

Received 22 July 2009 / Accepted 27 September 2010

\section{ABSTRACT}

\begin{abstract}
Aims. We present new spectropolarimetric observations of the chromospheric He I $10830 \AA$ multiplet observed in a filament during its phase of activity.

Methods. The data were recorded with the new Tenerife Infrared Polarimeter (TIP-II) at the German Vacuum Tower Telescope (VTT) on 2005 May 18. We inverted the He Stokes profiles using multiple atmospheric components.

Results. The observed He Stokes profiles display a remarkably wide variety of shapes. Most of the profiles show very broad Stokes $I$ absorptions and complex and spatially variable Stokes $V$ signatures. The inversion of the profiles shows evidence of different atmospheric blue- and redshifted components of the He I lines within the resolution element ( $1 \operatorname{arcsec})$, with supersonic velocities of up to $\sim 100 \mathrm{~km} \mathrm{~s}^{-1}$. Up to five different atmospheric components are found in the same profile. We show that even these complex profiles can be reliably inverted.
\end{abstract}

Key words. Sun: chromosphere - magnetic fields - Sun: infrared

\section{Introduction}

Filaments are typically elongated dark structures on the solar disk, characterized by additional absorption in strong chromospheric lines, like the $\mathrm{H} \alpha$ line. They are the on-disc counterparts of prominences seen above the solar limb and represent dense and cool chromospheric gas suspended in the hot and thin corona. The strength and structure of the magnetic field plays a key role in keeping the prominence material from flowing down to normal chromospheric levels. It is therefore of considerable interest to determine the magnetic structure associated with a prominence. A number of observations of the magnetic vector in prominences have been carried out (e.g., Leroy 1989; Casini et al. 2003; Lin et al. 1998; Trujillo Bueno et al. 2002; Merenda et al. 2006; Kuckein et al. 2009). All these investigations, except for the last one, have been restricted to quiescent prominences.

In this paper we introduce spectropolarimetric observations in the He I $10830 \AA$ triplet of a filament located in an active region that has been activated by a flare. Filaments also appear as dark absorption features in the He I $10830 \AA$ line.

Spectropolarimetry in the He I triplet at $10830 \AA$ is an important tool to determine the magnetic field vector in the solar chromosphere (Trujillo Bueno et al. 2002; Solanki et al. 2003). It is well suited for the measurement of the magnetic vector near the base of the solar corona because these lines are often narrow, nearly optically thin, have a reasonable effective Landé factor (see Table 1) and are easily observed (Rüedi et al. 1995). Measurements of the polarization of the He I $10830 \AA$ radiation are also indicated as a useful new tool for the diagnostics of the magnetic field in filaments (Lin et al. 1998; Trujillo Bueno et al. 2002). Recently, Kuckein et al. (2009) studied the vector magnetic field of an active region filament by analyzing spectropolarimetric data in the He I $10830 \AA$ lines with three different methods, one of these being, as in our case, MilneEddington inversions. They find the highest field strengths measured in filaments so far, around 600-700 G, and conclude that strong transverse magnetic fields are present in active region filaments.

The He I $10830 \AA$ multiplet originates between the atomic levels $2{ }^{3} \mathrm{~S}_{1}$ and $2{ }^{3} \mathrm{P}_{2,1,0}$. It comprises a component at $10829.0911 \AA$ with $J_{\mathrm{u}}=0$ (hereafter referred to as $\operatorname{Tr} 1$ ), and two components at $10830.2501 \AA$ with $J_{\mathrm{u}}=1(\operatorname{Tr} 2)$ and at $10830.3397 \AA$ with $J_{\mathrm{u}}=2(\operatorname{Tr} 3)$ which are blended at solar chromospheric temperatures. In the subsequent discussion we call this blended absorption the $\operatorname{Tr} 2,3$ component.

We concentrate on describing the observations and the inversion of these profiles. The He Stokes profiles in the activated filament reveal a remarkable level of complexity, requiring up to five independent magnetic components to reproduce. In a following paper the obtained maps of the magnetic and velocity fields in the filament will be presented, critically analyzed and interpreted in terms of prominence models.

\section{Observations}

On 2005 May 18 we observed the active region NOAA 10763 , located at $24^{\circ} \mathrm{W}, 14^{\circ} \mathrm{S}$ on the solar disk, which corresponds to a cosine of the heliocentric angle, $\mu=0.9$. During the observations a flare of GOES class C2.0 erupted in the western part of the active region. A filament was present in the active region close to and partly overlying the flare ribbons, which was activated by the flare.

The analyzed data were recorded with the second generation Tenerife Infrared Polarimeter TIP-II (Collados et al. 2007) mounted at the $70 \mathrm{~cm}$ aperture Vacuum Tower Telescope (VTT) at the Teide observatory in Tenerife. The TIP-II instrument allows for a spectral window of $11 \AA$ around the He $10830 \AA$ line 
Table 1. Spectral lines contained in the spectral window of the TIP-II instrument (10 825-10 $836 \AA$ ).

\begin{tabular}{lcc}
\hline \hline Line & Wavelength $(\AA)$ & Effective Landé factor \\
\hline $\mathrm{Si} \mathrm{I}$ & 10827.09 & 1.5 \\
$\mathrm{He}$ I (Tr1) & 10829.09 & 2.0 \\
$\mathrm{Ca}$ I & 10829.27 & 1.00 \\
$\mathrm{He}$ I (Tr2) & 10830.25 & 1.75 \\
$\mathrm{He}$ I (Tr3) & 10830.34 & 1.25 \\
$\mathrm{H}_{2} \mathrm{O}$ (telluric) & 10832.11 & \\
$\mathrm{Ca}$ & 10833.38 & 1.00 \\
$\mathrm{H}_{2} \mathrm{O}$ (telluric) & 10833.90 & \\
$\mathrm{Na}$ & 10834.85 & 1.03 \\
\hline
\end{tabular}

to be recorded with a wavelength dispersion of $11 \mathrm{~m} \AA$ per pixel. The wide wavelength range turned out to be absolutely necessary to cover the full range of possible He absorption signatures observed during the evolution of the filament. As we shall show below in Sect. 4, some of the He I profiles we observed display absorption at redshifts corresponding to as much as $100 \mathrm{~km} \mathrm{~s}^{-1}$. This is considerably higher than the highest redshifts measured by Aznar Cuadrado et al. (2005), whose exhaustive analysis of supersonic downflows seen in the He I lines was limited by the spectral range available to the much smaller TIP-I detector (Martínez Pillet et al. 1999).

The spectral window of TIP-II (10 825-10836 ̊) contains photospheric lines of Si I at 10827.09 $\AA$, Ca I at 10829.27 and $10833.38 \AA$ and Na I at $10834.85 \AA$, the chromospheric He I multiplet and two telluric blends at $10832.11 \AA$ and $10833.90 \AA$ (see Table 1).

The filament was scanned in steps of $0.35^{\prime \prime}$ perpendicular to the slit orientation $\left(180.51^{\circ}\right.$ with respect to the solar N-S direction), from 14:38:29 to 15:02:26 UT, providing a map of the region of $36.5 \times 25 \mathrm{Mm}^{2}$.

Figure 1 shows Stokes $I$ maps of the observed region obtained in the continuum at $10825.8 \AA$ (a) and in the core of the $\operatorname{Tr} 2,3$ component of the He I triplet (b). The slit is oriented along the $y$-axis and the direction of the scan is along the $x$-axis. Therefore, along the $x$-axis both spatial and temporal information are mixed. In addition to a few small pores, Fig. 1a clearly shows the granulation across the whole image, suggesting that the seeing conditions during the scan were particularly good and stable, allowing on average a resolution of 1 ". The resolution was determined by performing a Fast Fourier Transform of the average continuum image. Note that the diffraction-limited performance of the VTT at this wavelength corresponds to a resolution of $0.39^{\prime \prime}$. At some locations the He I profile is so broad that no pure continuum is present on the detector. These locations appear as dark cloud-like features in Fig. 1a. In Fig. 1b a strong absorption from the filament material is clearly visible in the He I lines at $9^{\prime \prime}<x<33^{\prime \prime}$, scanned from $\sim 14: 43$ to 14:53 UT.

In the last part of the map $\left(33^{\prime \prime}<x<49^{\prime \prime}\right)$, at $y \approx 19^{\prime \prime}-28^{\prime \prime}$, during the scan of a flare ribbon, the absorption in the He I lines is weaker than in quiet Sun regions and the spectra also show in part emission profiles (visible as brightenings in the image; cf. Lagg et al. 2007). In this region the $\mathrm{H} \alpha$ slit-jaw images that were simultaneously recorded at the VTT display flare ribbons as well.

\section{Analysis of the Stokes profiles}

In Fig. 2 four observed Stokes I profiles normalized to the continuum intensity are plotted as an example of the unusually wide range of profiles observed. These spectra and all the spectra we show here are binned over four spectral pixels (resulting in a wavelength dispersion of $44 \mathrm{~m} \AA$ ) and eight spatial pixels (resulting in a spatial pixel size of $0.7 \times 0.7 \operatorname{arcsec}^{2}$, a single pixel being $0.35 \times 0.18 \operatorname{arcsec}^{2}$ ) in order to increase the signal-to-noise ratio to $\sim 4000$. The solid line represents a profile observed in a comparatively quiet region of the map (at the location of the asterisk in Fig. 1b), the dashed spectrum shows a He I profile in emission observed in a flare ribbon (cross in Fig. 1b). Note that the Si I line is weakened at this location. The dash-dotted line (triangle in Fig. 1b) shows a very broad absorption in the He I lines observed in the filament. Significant absorption covers the wavelength range from $10829 \AA$ to $10835 \AA$, with weak absorption to the very edge of the detector at $10836 \AA$. The Stokes $I$ profile exhibits multiple minima that are not corresponding to the rest wavelengths of lines normally found in the Sun's spectrum, nor to telluric absorptions. The multiple absorption dips in the profile suggests multiple atmospheric components with a shift in the He I absorption caused by different velocities. For the dash-dotted profile these components are nearly at rest or are redshifted (see also Fig. 3). The maximum redshift corresponds to a downflow velocity along the line-of-sight of $\sim 100 \mathrm{~km} \mathrm{~s}^{-1}$. The dotted line (square in Fig. 1b) is an example of a particularly deep absorption in the He I line, which becomes as deep as the strong Si I line in the quiet Sun. In addition to this strong unshifted absorption there is a broad blueshifted absorption that blends with the Si I $10827 \AA$ line and extends even bluewards of it, revealing rapidly upward propagating chromospheric gas. The maximum blueshift corresponds to a velocity along the line-ofsight of $\sim 60 \mathrm{~km} \mathrm{~s}^{-1}$. As we already pointed out, the shown spectra are always binned, but multiple components are also seen in the single profiles forming the composite.

In Fig. 3 we plot the (averaged) full Stokes vector for the dash-dotted profile of Fig. 2 (position $x=32^{\prime \prime}, y=22^{\prime \prime}$ in Fig. 1). The polarized Stokes parameter displaying the highest signal-to-noise ratio is Stokes $V$. It displays a significant signal over nearly the entire wavelength range over which Stokes $I$ exhibits a strong absorption. The complexity of the Stokes $V$ profile confirms that the broad He I absorption is most likely caused by multiple atmospheric components harboring gas that flows at different speeds. It also indicates that at least a part of the downflowing gas is located in magnetized regions of the solar atmosphere. Stokes $Q$ and, to a lesser extent, Stokes $U$ also display a signal over a broad wavelength range, although the signal is weak and consequently the signal-to-noise ratio is fairly low. In Fig. 3 the wavelength positions at rest of the different lines present in the observed wavelength range are indicated by the vertical dotted lines (see also Table 1).

In order to investigate how the multiple He components are related with the motion of the filament material, we show in Fig. 4 two intensity maps of the scanned active region at wavelength positions $-1.08 \AA$ (A) and $+1.08 \AA$ (B) with respect to the rest wavelength of the $\operatorname{Tr} 2,3$ component of the He lines. The wavelengths at which the images are shown in Fig. 4 correspond to up- and downflows of $30 \mathrm{~km} \mathrm{~s}^{-1}$, respectively, and are marked by arrows in Fig. 2. From these maps we can see that strong blueor redshifted line profile components are present over practically the whole filament, suggesting strong dynamics throughout this active filament. These dynamics can be very complex, because we note a number of locations at which profiles with strong blueand redshifts coexist within a resolution element. Consequently, upflows and downflows are present at the same spatial position, although with this simple analysis alone we cannot rule out 

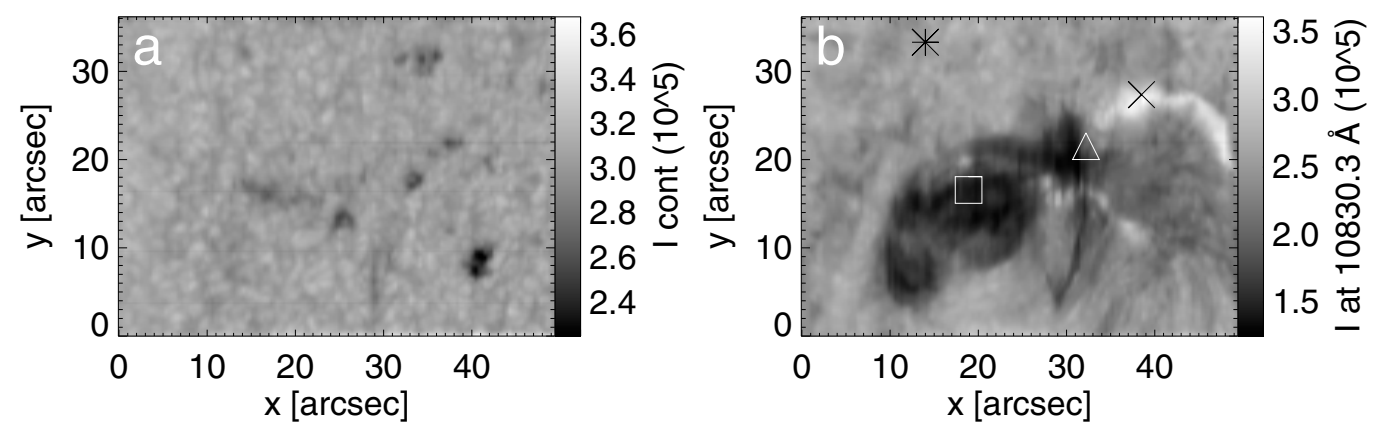

Fig. 1. Intensity maps at different wavelengths of the scanned portion of active region NOAA $10763\left(24^{\circ} \mathrm{W}, 14^{\circ} \mathrm{S}\right)$. Left a): continuum. Right $\left.\mathbf{b}\right)$ : He I line core of the Tr2,3 component. The four symbols overplotted on the right image refer to the line profiles shown in Fig. 2.

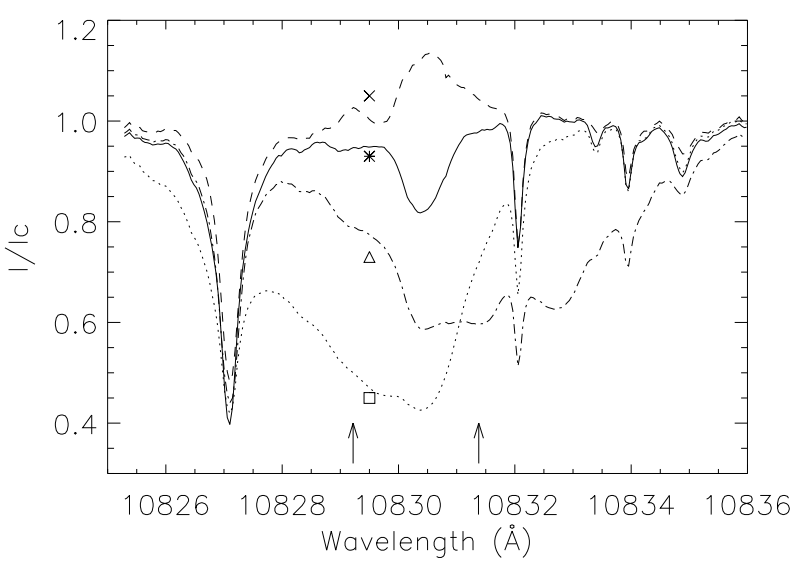

Fig. 2. Observed Stokes $I$ profiles at different locations, indicated by the different symbols in Fig. 1b. Solid line (star symbol): quiet Sun profile. Dashed line (cross): He I emission profile. Dash-dotted line (triangle): very broad He I profile indicating multiple redshifted atmospheric components. Dotted line (square): deep absorption in the He lines, that is in part strongly blueshifted. The arrows mark the wavelengths at which images are shown in Fig. 4.

that these profiles are simply strongly turbulently broadened, or a strong $\operatorname{Tr} 1$ mimics a blueshift. Indeed, a similar anomalous broadening was observed by López Ariste \& Casini (2006) in the $\mathrm{He}_{\mathrm{ID}}$ line in spicules. They found that a distribution of velocities with $F W H M=50 \mathrm{~km} \mathrm{~s}^{-1}$ was required to reproduce the observed broadening.

In Fig. 5 we present some examples of observed averaged Stokes $I$ (A) and Stokes $V$ (B) profiles at different positions marked by the letters a-f in Fig. 4. They stretch approximately from one end of the filament to the other and display a gradual change from mainly redshifted (a) to mainly blueshifted (f) profiles. All these profiles are characterized by multiple atmospheric components of the He I lines. In some of them, most prominently in $\mathrm{c}$ and e, blueshifted and redshifted components coexist. Note also the changing ratios of the Si I and the He I Stokes $V$ profile amplitudes. At some locations, notably b and c, the photospheric and chromospheric Stokes $V$ profiles have opposite polarities. This indicates that the magnetic structure changes quite dramatically between the photosphere and the chromosphere, which is not unusual for filaments (Leroy 1989; Low \& Zhang 2002). We also have evidence of different He components showing emission and absorption within the same profile (not shown in this paper), which are generally co-located with the bright $\mathrm{H} \alpha$ flare kernels, but do not necessarily appear bright in Fig. 1 b.

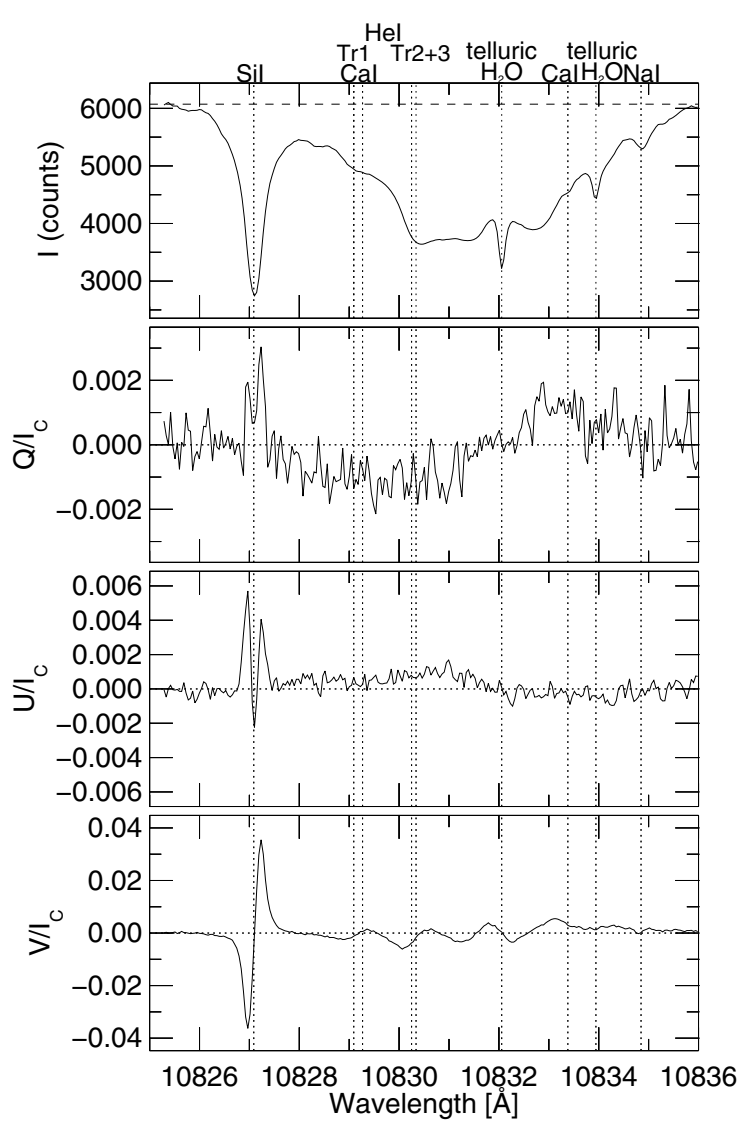

Fig. 3. Example of an observed averaged Stokes vector (dash-dotted profile of Fig. 2). The unusually broad Stokes I profile and the corresponding signatures of the $V$ profile suggest that the He triplet samples multiple atmospheric components. The vertical dotted lines mark the rest wavelengths of the different lines that are present in the observed wavelength range and are identified at the top of the figure and listed in Table 1.

\subsection{Stokes inversion}

We analyze the spectropolarimetric observations with the numerical code HELIX ${ }^{1}$ for the synthesis and inversion of Stokes profiles in a Milne-Eddington atmosphere (Lagg et al. 2004) extended to include the Paschen-Back effect (Sasso et al. 2006; Socas-Navarro et al. 2004). HELIX allows for inversions of a single spectrum using multiple atmospheric components, so that

1 The inversion code HELIX is freely available for the solar community. Please contact A. Lagg to obtain a copy. 

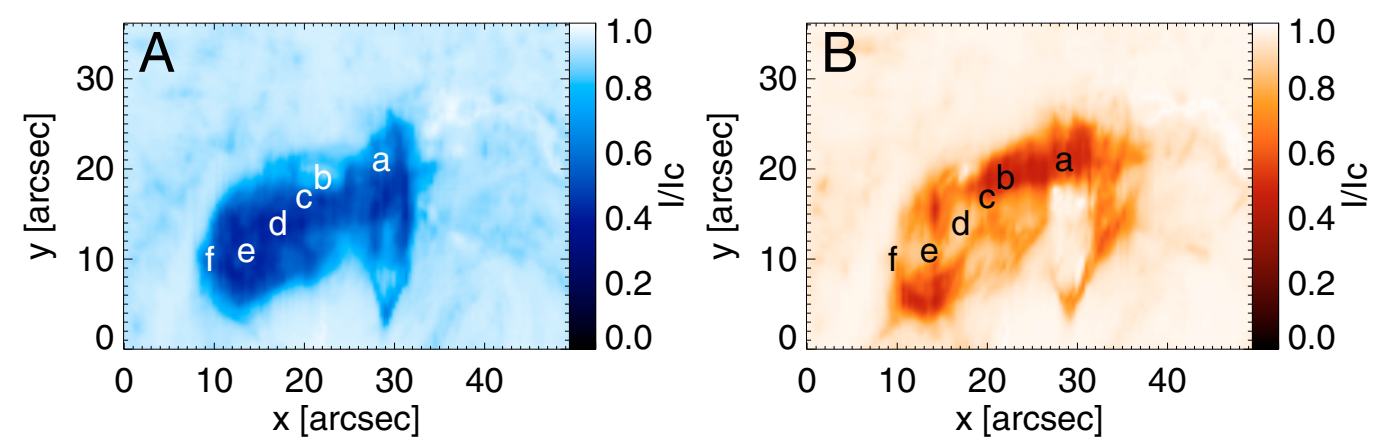

Fig. 4. Intensity maps of the scanned active region obtained at different wavelength positions indicated by arrows in Fig. 2. Left $\mathbf{A}$ ): At $-30 \mathrm{~km} \mathrm{~s}{ }^{-1}$ with respect to the central rest position of the Tr2,3 component of the He triplet. Right $\mathbf{B}$ ): at $+30 \mathrm{~km} \mathrm{~s}^{-1}$ with respect to the central rest position of the $\operatorname{Tr} 2,3$ component of the He triplet. The different letters refer to the line profiles shown in Fig. 5.

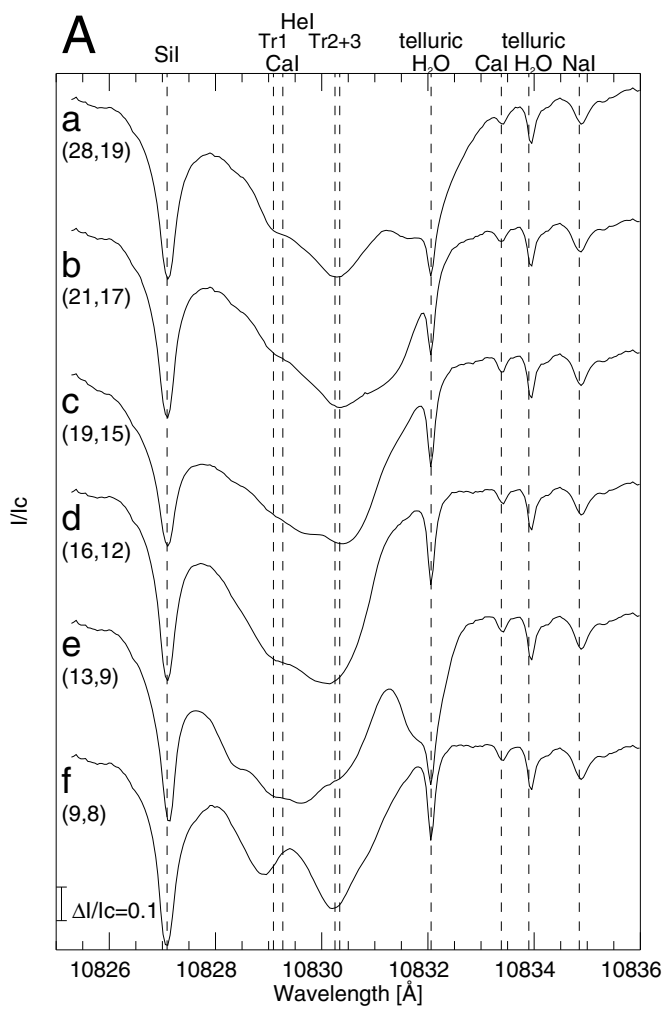

atmospheric parameters like the magnetic field vector and the line-of-sight (LOS) velocity are retrieved for each component. The free parameters tuned by the code to fit the observed Stokes profiles are the magnetic field strength $B$ and direction (inclination angle $\gamma$ and azimuthal angle $\chi$ ), the line-of-sight velocity $v_{\mathrm{LOS}}$, the Doppler width $\Delta \lambda_{\mathrm{D}}$, the damping constant $\Gamma$, the ratio of the line center to the continuum opacity $\eta_{0}$ and the gradient of the source function $S_{1}$. For more information on these parameters we refer to Lagg et al. (2004). A range for every parameter to be fitted is specified to guarantee that the result of the inversion stays within the regime of physically useful solutions.

The inversion includes a scattering polarization correction, a treatment of the Hanle effect for a special case. It is only valid at disk center and for horizontal fields and it only improves the azimuth retrieval (Trujillo Bueno et al. 2002). Indeed, in the particular case of a LOS-direction perpendicular to the solar surface (disk center) and a magnetic field direction parallel to the solar surface (horizontal field) the polarization signal owing to the Hanle effect is oriented along the magnetic field direction: $\tan (2 \chi)=(U / Q)$.
Fig. 5. Observed averaged Stokes $I \mathbf{A})$ and $V \mathbf{B})$ profiles at the locations marked by letters in Fig. 4. The dashed, vertical lines mark the central position of the spectral lines in the wavelength field-of-view of the TIP-II instrument. The $(x, y)$ coordinates in arcseconds are written next to each profile.

The details of the input atmospheres depend on the number of He components necessary to reproduce the observed profiles. One parameter, the filling factor $\alpha_{i}$, defines the contribution of a given atmospheric component $i$ to the total observed He profile. The sum of the filling factors of all atmospheric components within a resolution element is required to be unity, $\sum_{i} \alpha_{i}=1$. The maximum number of $\mathrm{He}$ atmospheric components we need to reproduce the profiles in the scan is five (see Fig. 6). This is considerably more than in previous studies involving the $\mathrm{He}$ line, and we need to demonstrate that these fits are reliable. Additionally, we need to determine the maximum amount of information that can be reliably gathered from these complex inversions.

We have tested that we do not employ more components than necessary by first fitting each profile with a smaller number of atmospheric components and consequently free parameters. Only a significant improvement of the fitness function $f$ (defined in Lagg et al. 2004), which gives the goodness of the fit, warrants the use of more components or free parameters. Usually, an insufficient number of free parameters results in a poor fit for parts of the observed line profile. 
For some extreme profiles we face the problem of a huge number of free parameters to be fitted. The spectral region covered by the $\mathrm{He}$ absorption signature of strongly broadened or shifted profiles also contains four photospheric lines of Si I, Ca I (two lines) and $\mathrm{NaI}$ and two telluric blends (see Table 1). We have to fit these lines as well to obtain unbiased fits of the He I absorption, which often blends with some or all of these lines. Because the photospheric magnetic and velocity vector in general significantly differs from the chromospheric parameters, this increases the total number of atmospheric components and requires additional line-specific parameters of each line to be determined. For simplicity, each spectral line was assigned its own atmospheric component. The number of free parameters was limited by coupling them appropriately (see Sect. 3.2). To obtain a good fit of the strong photospheric Si I line at $10827.09 \AA$, we had to consider two atmospheric components: a magnetic component and a field-free stray-light component. Only this combination could satisfactorily reproduce both the line core and wings of the Si I line. The two telluric blends result in eight additional free parameters, originating from fitting two Voigt profiles to the telluric lines. The parameters needed to fit the telluric blends could be kept in a very narrow range over the whole map, introducing no decrease in stability of the convergence of the inversion.

There are profiles in the scan where the He lines show emission features. These profiles were excluded from further analysis. Even though the HELIX code can fit emission profiles by setting the gradient of the source function $S_{1}$ to a negative number, the analysis technique is not optimized to produce reliable results. Moreover, in some spatial pixels the He emission profiles coexist with blue- or redshifted He absorptions, making the retrieval even more complicated. In the map shown in Fig. 6, the pixels containing profiles that were not inverted are black.

\subsection{Parameter coupling}

In order to decrease the number of free parameters and thus to enhance the stability of the fitting procedure, we coupled some atmospheric parameters between the atmospheric components, so that a given atmospheric parameter was forced to have the same (but not prescribed) value in all atmospheric components. Coupling between parameters of different components was also used as a means to impose that all lines from the same atmospheric layer (in particular the photosphere) must be formed in the same atmosphere. We also narrowed the ranges of possible solutions for some other parameters, which also helped to ensure the uniqueness of the solution.

In particular, we coupled the magnetic field vector $(B, \gamma$ and $\chi$ ) and the gradient of the source function $S_{1}$ between the four photospheric lines, assuming that their values stay approximatively constant. This is consistent with the assumption that the four lines are formed at similar heights and can be represented by a single atmosphere.

Among the photospheric lines, the Si I line is the strongest one with the best signal-to-noise ratio and shows clear signatures in the Stokes profiles. Changes in the atmospheric parameters will have a larger influence on the Stokes profiles of the $\mathrm{Si}$ line than on the weaker photospheric lines. Therefore, the photospheric parameters are mainly determined by the shape and strength of the Si Stokes profiles. Then, we made an inversion run for the whole map, fitting only the photospheric and the telluric lines. From the results of the fit, assuming that these lines are not disturbed by the filament activity happening at higher

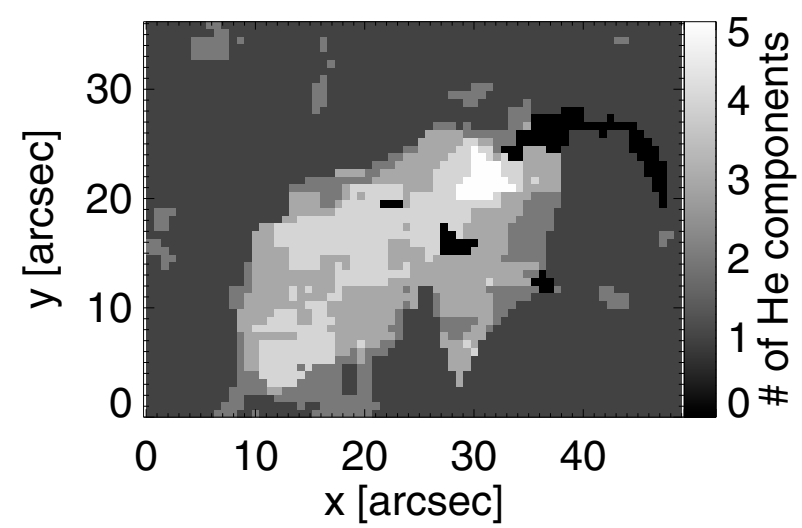

Fig. 6. Number of He components needed to fit the observed profiles for each pixel of the map. In the map, the pixels containing profiles that were not inverted are black (mainly those in which the He profile is in emission).

Table 2. Values of the fitness functions $f$ returned by the fits to 25 observed averaged profiles.

\begin{tabular}{cccccc}
\hline \hline $\begin{array}{c}\text { Pixel x/y } \\
(\operatorname{arcsec})\end{array}$ & $\begin{array}{c}78-79 \\
(27.3)\end{array}$ & $\begin{array}{c}80-81 \\
(28.0)\end{array}$ & $\begin{array}{c}82-83 \\
(28.7)\end{array}$ & $\begin{array}{c}84-85 \\
(29.4)\end{array}$ & $\begin{array}{c}86-87 \\
(30.1)\end{array}$ \\
\hline $104-107$ & $f_{1}=1.44$ & 1.42 & 1.53 & 1.54 & 1.29 \\
$(17.7)$ & $f_{2}=1.48$ & 1.71 & 1.75 & 1.58 & 1.17 \\
& $f_{3}=1.15$ & 1.46 & 1.29 & 1.39 & 1.16 \\
\hline $108-111$ & $f_{1}=1.95$ & 1.81 & 1.74 & 1.86 & 1.18 \\
$(18.4)$ & $f_{2}=1.91$ & 1.55 & 1.76 & 1.77 & 1.04 \\
& $f_{3}=1.67$ & 1.48 & 1.48 & 1.69 & 1.08 \\
\hline $112-115$ & $f_{1}=1.89$ & 1.68 & 1.73 & 1.25 & 1.50 \\
$(19.0)$ & $f_{2}=2.00$ & 1.63 & 1.60 & 1.28 & 0.94 \\
& $f_{3}=1.78$ & 1.53 & 1.34 & 1.23 & 1.35 \\
\hline $116-119$ & $f_{1}=1.59$ & 1.76 & 1.41 & 1.58 & 1.56 \\
$(19.7)$ & $f_{2}=1.47$ & 1.97 & 1.69 & 1.73 & 1.52 \\
& $f_{3}=1.32$ & 1.80 & 1.36 & 1.46 & 1.40 \\
\hline $120-123$ & $f_{1}=1.68$ & 1.98 & 1.51 & 1.54 & 1.76 \\
$(20.4)$ & $f_{2}=1.66$ & 1.88 & 1.51 & 1.38 & 1.60 \\
& $f_{3}=1.61$ & 1.92 & 1.53 & 1.43 & 1.58 \\
\hline
\end{tabular}

atmospheric layers, we could select a narrower input range for the Doppler width $\Delta \lambda_{D}$ of the photospheric lines and the width and the damping of the Voigt profile for the telluric blends. The narrower input ranges are then used for the inversion done including the He lines. This avoids that the code broadens and shifts these lines by large amounts to mimic one of the He components.

The different He components certainly have different LOS velocities, but it is not a priori clear if they sample different magnetic vectors (and if they do, whether there is sufficient information in the measured Stokes parameters to distinguish between different magnetic vectors affecting the various He I absorption components). Therefore, we made several test runs to decide whether to couple the magnetic field vector or not (see Sect. 4).

We also coupled the gradient of the source function $S_{1}$ between the He atmospheric components, and we could also assign a relatively narrow input range for $v_{\mathrm{LOS}}$ of each He component from the visual analysis of the observed profiles. Indeed, when the velocity separation between the He profiles is distinct enough $\left(\sim 10 \mathrm{~km} \mathrm{~s}^{-1}\right)$ we can clearly distinguish the positions of the different minima in the $I$ profile. 

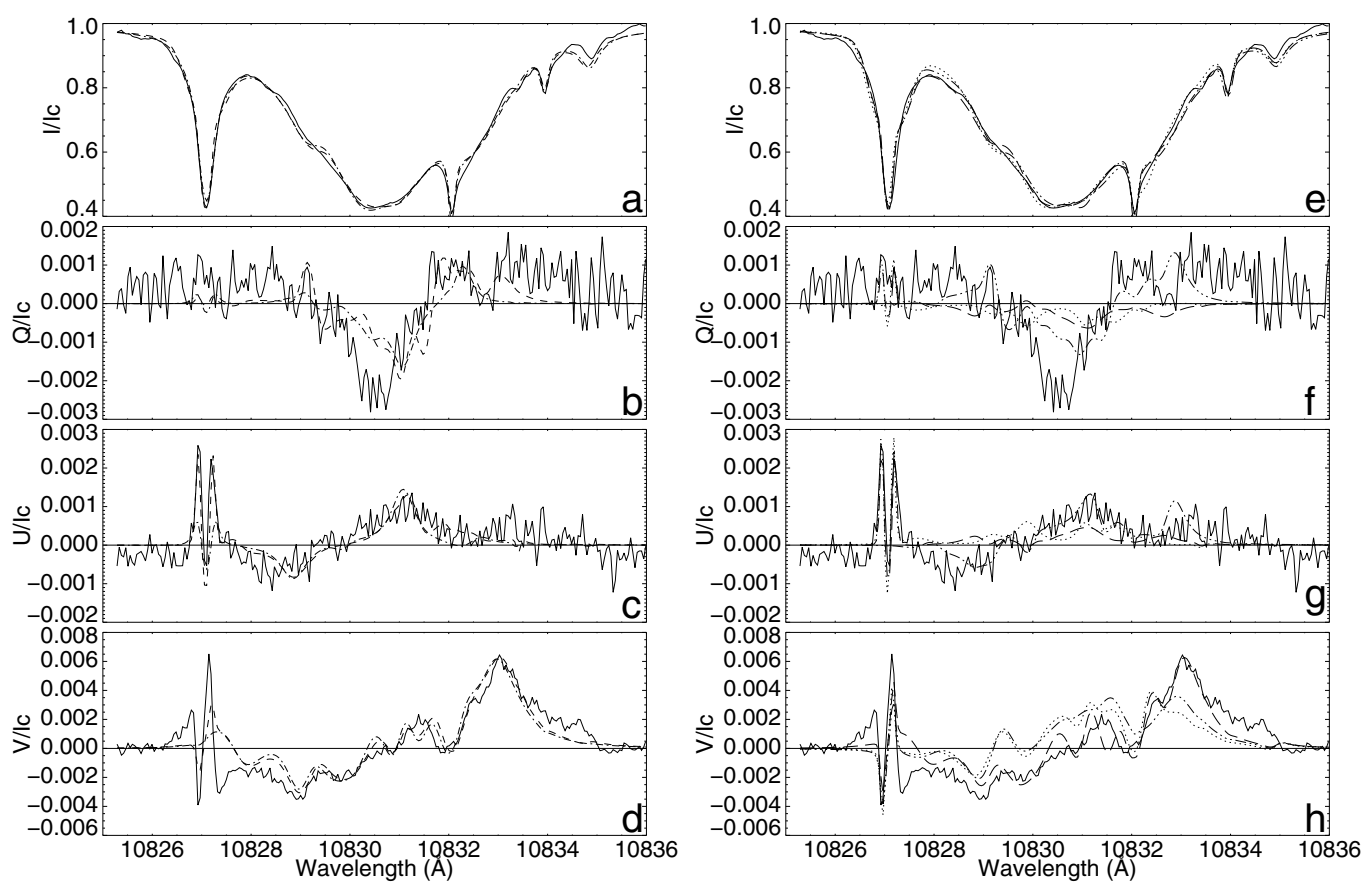

Fig. 7. Inversions of the Stokes $I, Q, U$, and $V$ averaged profiles at the location (29", 19") of the map in Fig. 1. The fits (dashed, dash-dotted, dotted, dash-dot-dotted and long dashed lines) to the observed profiles (solid lines) are obtained by allowing five different atmospheric components to describe the He I triplet. Left a)-d): the dashed profile is obtained by leaving the magnetic field vector free between the five He components while the dash-dotted profile is obtained by coupling only the magnetic field strength. Right $\mathbf{e}$ )-h): the dotted profile is obtained by coupling the magnetic field vector between the five He components, coupling the magnetic field strength and the inclination gives the dash-dot-dotted profiles, while coupling the field strength and the azimuth leads to the long dashed profiles.

\section{Results}

Test runs showed that the best fits to the observed Stokes profiles are obtained by coupling only the magnetic field strength $B$ between the He components, leaving the two angles, $\gamma$ and $\chi$, free. In Fig. 7 we show as an example the results of inversions of the observed averaged Stokes profiles (solid lines) at the location $\left(29^{\prime \prime}, 19^{\prime \prime}\right)$ of the map in Fig. 1. In Figs. 7a-d two fits to the observed profiles (dashed and dash-dotted lines) are displayed. Both are obtained by allowing five different atmospheric components to describe the He I lines. The dashed lines represent a fit obtained by allowing the code to find a different magnetic field vector for each atmospheric component used to describe the $\mathrm{He}$ profiles. For the fit represented by the dash-dotted lines though, we coupled the magnetic field strength between the components, while leaving the angles describing the magnetic field direction uncoupled. The average values of $f$ obtained from 100 inversion runs of this particular observed profile are 1.62 for the dashed line fit and 1.53 for the dash-dotted line fit. Both fits are good and the fitness values are comparable, but for the dash-dotted profile, the fit is obtained with 4 free parameters less. The slightly higher value of the fitness of the dashed profile is not enough to justify the use of so many more free parameters. In Figs. 7(e-h) the same observed profile (solid line) is plotted as in (a-d). The dotted line is obtained by coupling the whole magnetic field vector between the five components. The observed Stokes $Q, U$ and $V$ profiles cannot be reproduced by this inversion $(f=0.89)$. By coupling $B$ and $\chi$ (long dashed lines) we obtain a good fit only for $I$ and $V$ ( $f=1.26)$ and by coupling $B$ and $\gamma$ (dash-dot-dotted lines) we can fit $I, Q$, and $U$ reasonably well, but not $V(f=1.02)$. In no case can we reproduce the Stokes vector as a whole.

Stokes $Q$ of this observed profile shows a predominantly single-lobe signature that suggests resonance scattering polarization. The simple scattering polarization correction we used and described in Sect. 3.1 is not sufficient to fit the strong $Q$ peak. An improved treatment of the scattering polarization may help.

We carried out the same analysis for another 25 Stokes profiles with 3,4 , and $5 \mathrm{He}$ components in a region of $3.5 \times 1.8 \mathrm{Mm}^{2}$ around the profile displayed in Fig. 7. The values of the fitness function $f$ obtained from three different types of fits (leaving the magnetic field free, $f_{1}$, coupling $B, f_{2}$, and coupling $B$ and $\chi$, $f_{3}$ ) to the observed profiles are grouped in Table 2 . The profiles are located at the spatial pixels listed at the top (x) and on the left (y) of Table 2. These observed profiles can be reproduced almost equally well either by coupling the magnetic field strength between the He components or by leaving the full magnetic field vector free. Significantly poorer fits to the full Stokes vectors are obtained by coupling $B$ and $\chi$. Indeed, $f_{3}<f_{1}$ in $88 \%$ of the cases and $f_{3}<f_{2}$ in $80 \%$ of the cases. $f_{1}$ is higher than $f_{2}$ in $56 \%$ of the cases, while in over $40 \%$ of the cases $f_{2}>f_{1}$. The difference between the two is insignificant and the marginally higher value of $f_{1}$ is not enough to justify using a higher number of free parameters.

Another test is based on the analysis of the errors in the retrieval of the atmospheric parameters. The convergence of the genetic algorithm used for the minimization of the merit function, Pikaia (Charbonneau 1995), does not depend on the choice of initial guesses for the atmospheric parameters. The convergence to the global minimum occurs on a random path after an infinite number of iterations. We set an upper limit to the number of iterations (700) in a way that the necessary computing time and the stability of the resulting fitness, $f$, are in good balance. The variations of the parameter values resulting from numerous inversion runs with an upper limit to the number of iterations can be used as a direct measure of the error in the retrieval of 
C. Sasso et al.: Multicomponent He I $10830 \AA$ profiles in an active filament

Table 3. Mean values of the atmospheric parameters with the errors returned by the fits displayed in Fig. 7.

\begin{tabular}{|c|c|c|c|c|c|}
\hline Parameter/Fit-profile & $\begin{array}{c}\text { Dashed } \\
\text { No coupling }\end{array}$ & $\begin{array}{c}\text { Dash-dotted } \\
B \text { coupled }\end{array}$ & $\begin{array}{c}\text { Dash-dot-dotted } \\
B, \gamma \text { coupled }\end{array}$ & $\begin{array}{l}\text { Long dashes } \\
B, \chi \text { coupled }\end{array}$ & $\begin{array}{c}\text { Dotted } \\
B, \gamma, \chi \text { coupled }\end{array}$ \\
\hline$B \pm \Delta B(\mathrm{G})$ & $\begin{array}{l}478 \pm 330 \\
264 \pm 215 \\
403 \pm 285 \\
307 \pm 159 \\
632 \pm 376\end{array}$ & $228 \pm 53$ & $250 \pm 134$ & $234 \pm 55$ & $222 \pm 123$ \\
\hline$\gamma \pm \Delta \gamma\left({ }^{\circ}\right)$ & $\begin{array}{c}47 \pm 25 \\
122 \pm 22 \\
108 \pm 24 \\
131 \pm 21 \\
141 \pm 22\end{array}$ & $\begin{array}{c}33 \pm 22 \\
110 \pm 4 \\
102 \pm 7 \\
141 \pm 17 \\
170 \pm 10\end{array}$ & $122 \pm 23$ & $\begin{array}{c}31 \pm 26 \\
110 \pm 5 \\
102 \pm 7 \\
143 \pm 17 \\
169 \pm 12\end{array}$ & $124 \pm 22$ \\
\hline$\chi \pm \Delta \chi\left(^{\circ}\right)$ & $\begin{array}{c}-29 \pm 34 \\
46 \pm 70 \\
58 \pm 28 \\
7 \pm 16 \\
8 \pm 33 \\
\end{array}$ & $\begin{array}{c}-18 \pm 48 \\
52 \pm 62 \\
48 \pm 32 \\
0 \pm 13 \\
-2 \pm 37\end{array}$ & $\begin{array}{c}-30 \pm 43 \\
60 \pm 53 \\
50 \pm 32 \\
1 \pm 13 \\
-3 \pm 37\end{array}$ & $30 \pm 42$ & $27 \pm 44$ \\
\hline$v_{\mathrm{LOS}} \pm \Delta v_{\mathrm{LOS}}\left(\mathrm{km} \mathrm{s}^{-1}\right)$ & $\begin{array}{c}-38 \pm 3 \\
1 \pm 1 \\
21 \pm 1 \\
53 \pm 1 \\
68 \pm 2\end{array}$ & $\begin{array}{c}-39 \pm 2 \\
1.1 \pm 0.7 \\
21 \pm 1 \\
53 \pm 1 \\
69 \pm 2\end{array}$ & $\begin{array}{c}-31 \pm 2 \\
0.9 \pm 1.0 \\
21 \pm 1 \\
53 \pm 1 \\
68 \pm 2\end{array}$ & $\begin{array}{c}-38 \pm 3 \\
1 \pm 1 \\
21 \pm 1 \\
53 \pm 1 \\
69 \pm 2\end{array}$ & $\begin{array}{c}-31 \pm 2 \\
0.9 \pm 1.0 \\
21 \pm 1 \\
53 \pm 1 \\
68 \pm 2\end{array}$ \\
\hline$\alpha \pm \Delta \alpha$ & $\begin{array}{l}0.10 \pm 0.03 \\
0.33 \pm 0.06 \\
0.20 \pm 0.05 \\
0.25 \pm 0.06 \\
0.12 \pm 0.05 \\
1.62 \pm 0.19\end{array}$ & $\begin{array}{l}0.10 \pm 0.02 \\
0.32 \pm 0.04 \\
0.19 \pm 0.03 \\
0.24 \pm 0.03 \\
0.14 \pm 0.03 \\
1.53 \pm 0.16\end{array}$ & $\begin{array}{l}0.09 \pm 0.02 \\
0.29 \pm 0.04 \\
0.19 \pm 0.04 \\
0.27 \pm 0.04 \\
0.16 \pm 0.04 \\
1.02 \pm 0.06\end{array}$ & $\begin{array}{l}0.10 \pm 0.03 \\
0.31 \pm 0.04 \\
0.21 \pm 0.03 \\
0.23 \pm 0.04 \\
0.15 \pm 0.03 \\
1.26 \pm 0.09\end{array}$ & $\begin{array}{l}0.10 \pm 0.03 \\
0.27 \pm 0.05 \\
0.21 \pm 0.03 \\
0.26 \pm 0.04 \\
0.16 \pm 0.03 \\
0.89 \pm 0.04\end{array}$ \\
\hline
\end{tabular}

this parameter. This error contains the intrinsic uncertainty in the parameter retrieval, caused by the similarity of Stokes profiles for different parameter values, and the error introduced by the inversion process itself.

The mean retrieved values of the atmospheric parameters with their errors, as deduced from 100 inversion runs, are listed in Table 3 for each of the profiles shown in Fig. 7. The five values per parameter correspond to the different He atmospheric components. As pointed out earlier both the dashed (no coupling) and the dash-dotted profiles (only $B$ coupled) provide almost equally good fits, but the latter is obtained by an inversion with a lower number of free parameters. The errors when $B$ is coupled are smaller than without coupling, in particular the errors on $B$ and $\gamma$. This means that with a lower number of free parameters the code is more stable. The $v_{\text {LOS }}$ is always well retrieved with a significantly lower error than the specified range for the $v_{\mathrm{LOS}}$ of any given component $\left(\sim 10 \mathrm{~km} \mathrm{~s}^{-1}\right)$. Both the magnetic field angles, $\gamma$ and $\chi$, can be determined for the individual components. The error in the inclination angle, $\gamma$, is sufficiently large that not all components can be clearly distinguished from each other (e. g. components 2 and 3 in Table 3), but the values obtained are in general good enough to deduce the polarity of the magnetic field. Regarding the azimuth, the signal-to-noise ratio in the $Q$ and $U$ profiles is barely high enough to reliably retrieve information on it.

The final three columns of Table 3 list the retrieved values with uncertainties for an increasing number of parameters that are forced to have the same value in all atmospheric components. Irrespective of whether we keep $B$ and $\gamma$ (dash-dot-dotted), $B$ and $\chi$ (long dashes) or $B, \gamma$ and $\chi$ (dotted) coupled, the fitness $f$ is significantly lower than if all parameters are uncoupled, or only $B$ is coupled. Furthermore, the spread (uncertainty) in the retrieved parameters tends to be larger in the last three columns, so that coupling additional parameters does not lead to an increase in the reliability of the retrieved solution, but exactly the opposite. Furthermore, the retrieved values of the coupled parameters are close to the $\alpha$-weighted averages of the uncoupled values of the same parameter. The exception is $B$, for which the coupled value is always lower. Based on the above test (and similar tests applied to the Stokes profiles in other pixels) we inverted all the observed profiles in the observed map by coupling the magnetic field strength between the He components, and leaving $\chi$ and $\gamma$ free (fitness $f_{2}$ in Table 2).

Examples of the inversions of increasingly complex He I $10830 \AA$ Stokes $I, Q, U$, and $V$ profiles are shown in Figs. 8-12. In each plot, the black lines are the averaged observed profiles, while the red lines are the best fits to the observations obtained considering one, two, three, four, and five atmospheric components of the He I lines. These are the minimum numbers of atmospheric components that are needed to obtain a satisfactory fit, both as judged by eye and from a decrease of $f$ found by adding a further component. Also plotted are the different $\mathrm{He}$ components (magenta, light blue, yellow, green, and dark gray lines), whose sum gives the red lines. The plotted contribution of each component is multiplied by its filling factor. In Table 4 we give the mean retrieved values of the atmospheric parameters $B, \gamma, \chi, v_{\mathrm{LOS}}$ and $\alpha$, together with their errors. The values listed in Table 4 are averages of the parameter values returned from 50 inversion runs, and the uncertainties reflect the spread (standard deviation) of the returned values. When multiple-component fits to the He line are required, such as those displayed in Figs. 9-12, the parameters retrieved for each component are given. Note that the photospheric lines are simultaneously fitted by two atmospheric components (one magnetic, one field free), as described in Sect. 3.1. For each fit we also give the value of the fitness function in the Table, $f \pm \Delta f$. 


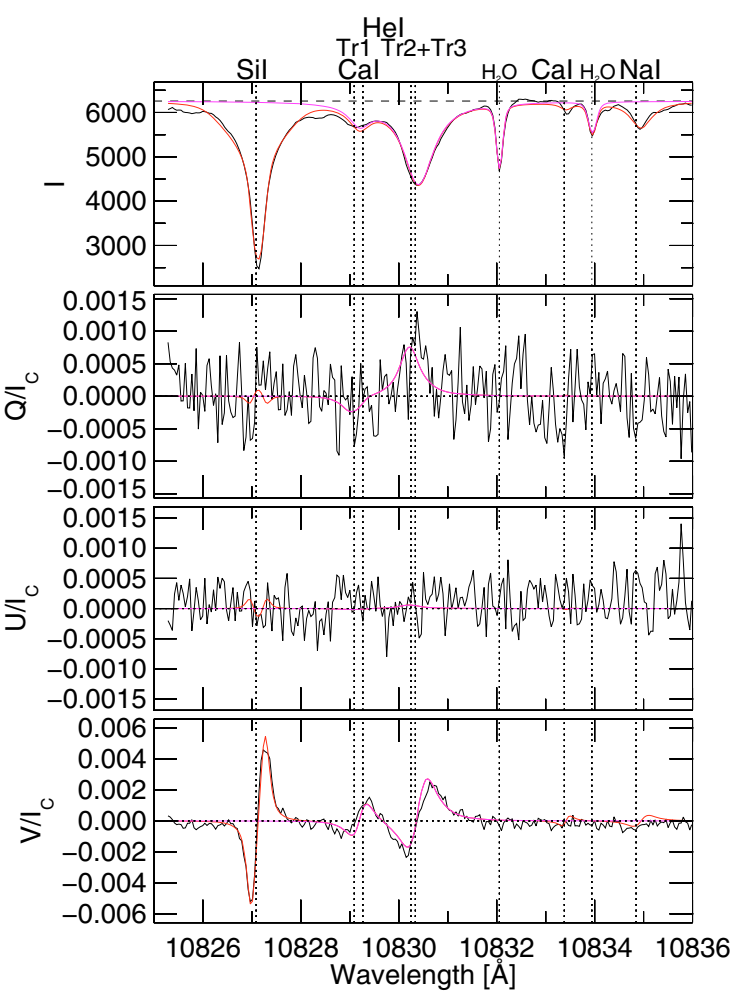

Fig. 8. Observed spatially averaged and inverted Stokes $I, Q, U$ and $V$ profiles at the location $\left(5^{\prime \prime}, 13^{\prime \prime}\right)$ of the map in Fig. 1. The solid black lines are the observed profiles, while the red lines are the fits to all observed lines obtained imposing one atmospheric component to describe the He I lines (magenta lines), and two for the photospheric lines.

Figure 8 displays a one-He-component fit to the observed Stokes profiles. Similar quiet profiles are found in the region of the scan outside the portion with the filament or other activity (flare). In Table 4 (first table) the mean values and the errors of the retrieved atmospheric parameters after 50 inversion runs are reported. Even with this simple profile we have difficulties in retrieving the values of the magnetic field angles, $\gamma$ and $\chi$, in particular $\chi$ with high accuracy. This is mainly because of the low signal-to-noise ratio of the observed $Q$ and $U$ profiles and to a smaller extent because of the already high number of free parameters to be fitted (the inversion includes the He, the photospheric and the telluric lines). Figures 9 and 10 show a two- and a three-component fit to other, more complex observed profiles. The two profiles, observed close in position and time, are somewhat similar, with the difference of a deeper He blueshifted absorption in the latter profile. This additional absorption requires an additional He component to be fitted. The presence of another He component in the profile of Fig. 10 is also evident from the different shape of the negative lobe in the Si I $V$ profile. In both profiles we find strongly supersonic blueshifted He components (speeds in excess of $-50 \mathrm{~km} \mathrm{~s}^{-1}$ ) coexisting with a He component nearby at rest. The fit to the observed profile shown in Fig. 11 requires an additional fourth redshifted He component. The final fit shown in Fig. 12 requires five atmospheric components. Only few other profiles (see Fig. 6) need five components to be reproduced, and these are all located around one end point of the observed filament. This is also the location where we measure the highest downflow velocities $\left(\sim 100 \mathrm{~km} \mathrm{~s}^{-1}\right)$. It is particularly gratifying to see that both the fitness $f$ and the uncertainties of the retrieved parameters appear to be nearly independent of the number of atmospheric components. This is

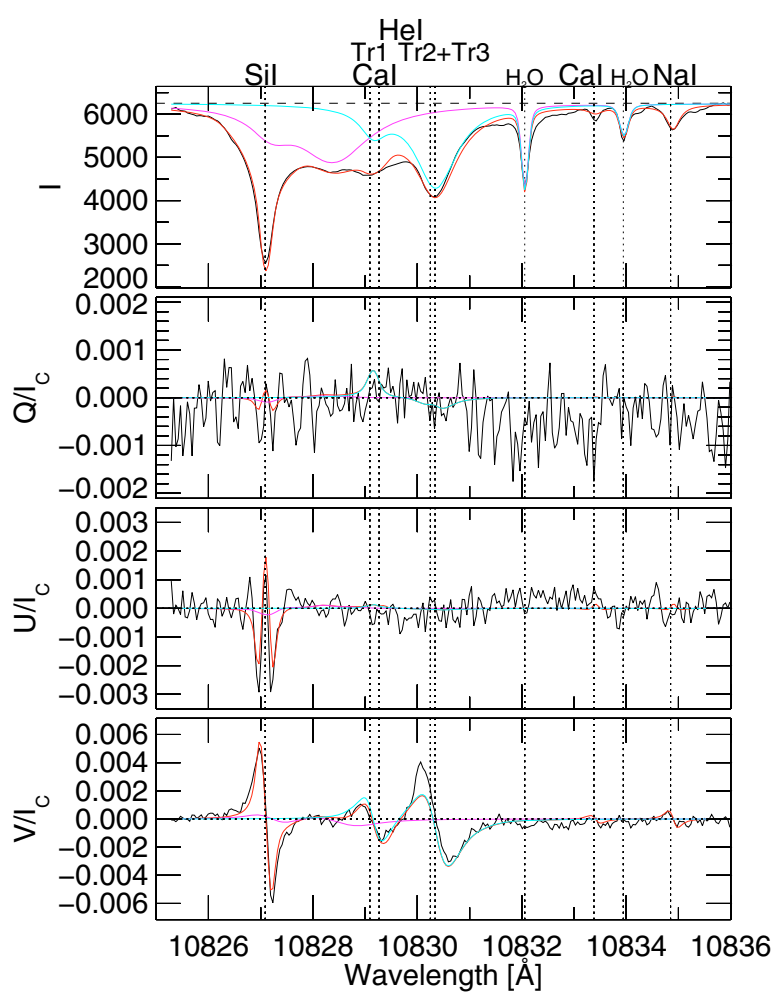

Fig. 9. Observed and inverted Stokes $I, Q, U$, and $V$ profiles at the location $\left(27^{\prime \prime}, 9^{\prime \prime}\right)$ of the map in Fig. 1. The solid black lines are the observed profiles, while the red lines are the fits obtained imposing two different atmospheric components to describe the He I lines (the profiles of the individual components are given by magenta and light blue lines).

probably because profiles with more components tend to have a larger equivalent width and individual components are well separated in wavelength.

\section{Discussion and conclusions}

We focus on the analysis and the inversion of Stokes profiles of the He I $10830 \AA$ triplet observed in an active region filament during its phase of activity. We showed that even the most complex observed profiles can be well reproduced by multi-component atmospheres. Although we cannot completely exclude that the profiles could be simply strongly turbulently broadened, the fits based on multiple components are of clearly better quality (see below). We tested the response of the numerical code HELIX for the inversion of Stokes profiles to work with a high number of atmospheric components and free parameters. This was necessary to fit not only the different observed $\mathrm{He}$ components, but also the photospheric and telluric lines in the wavelength range. We were able to retrieve the atmospheric parameters for the individual $\mathrm{He}$ components at different levels of accuracy. The values of the magnetic field strength, $B$, retrieved from the fit presented here are in the range 100-250 G. These values are lower than those found by Kuckein et al. (2009) for another active prominence. This difference could be caused by the different methods employed, because unlike Kuckein et al. (2009) we inverted our profiles including a scattering polarization correction (see Sect. 3.1) and the magnetic field strength we retrieved is a result of a coupling between many He components. The difference could also be merely a reflection of the different strengths of the two filaments' magnetic fields, or the analyzed profiles possibly do not sample the highest field strength 


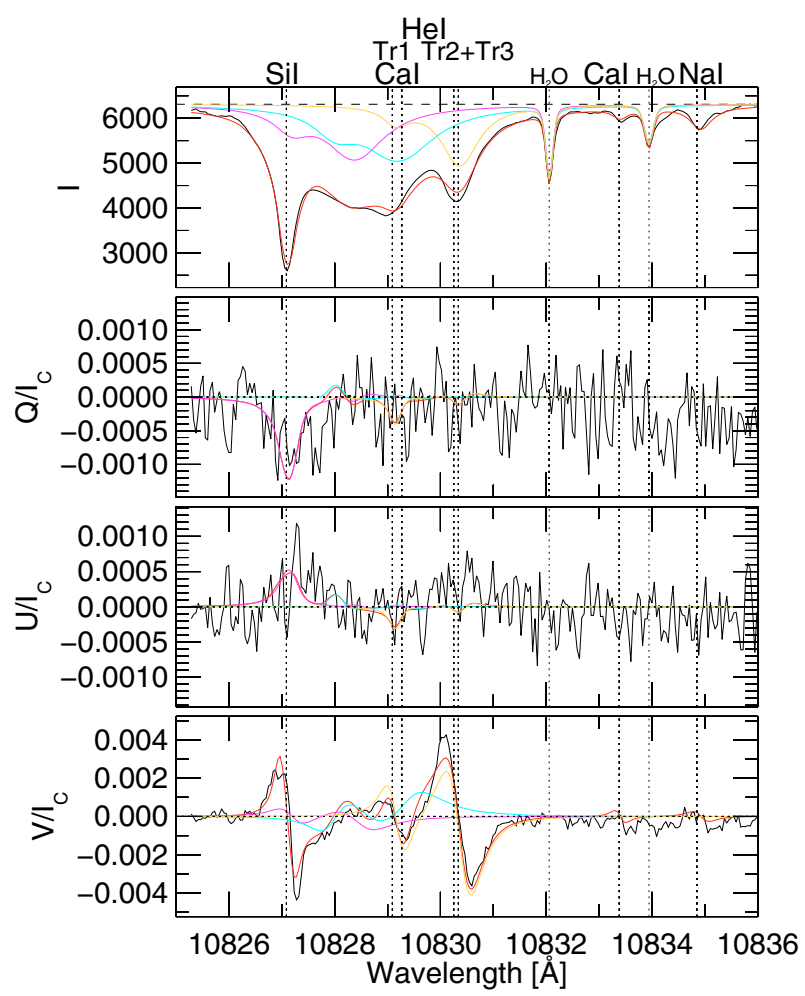

Fig. 10. Observed and inverted Stokes $I, Q, U$, and $V$ profiles at the location $\left(29^{\prime \prime}, 13^{\prime \prime}\right)$ of the map in Fig. 1. The solid black lines are the observed profiles, while the red lines are the fits obtained by allowing three different atmospheric components to describe the He I lines (represented by magenta, light blue, and yellow lines).

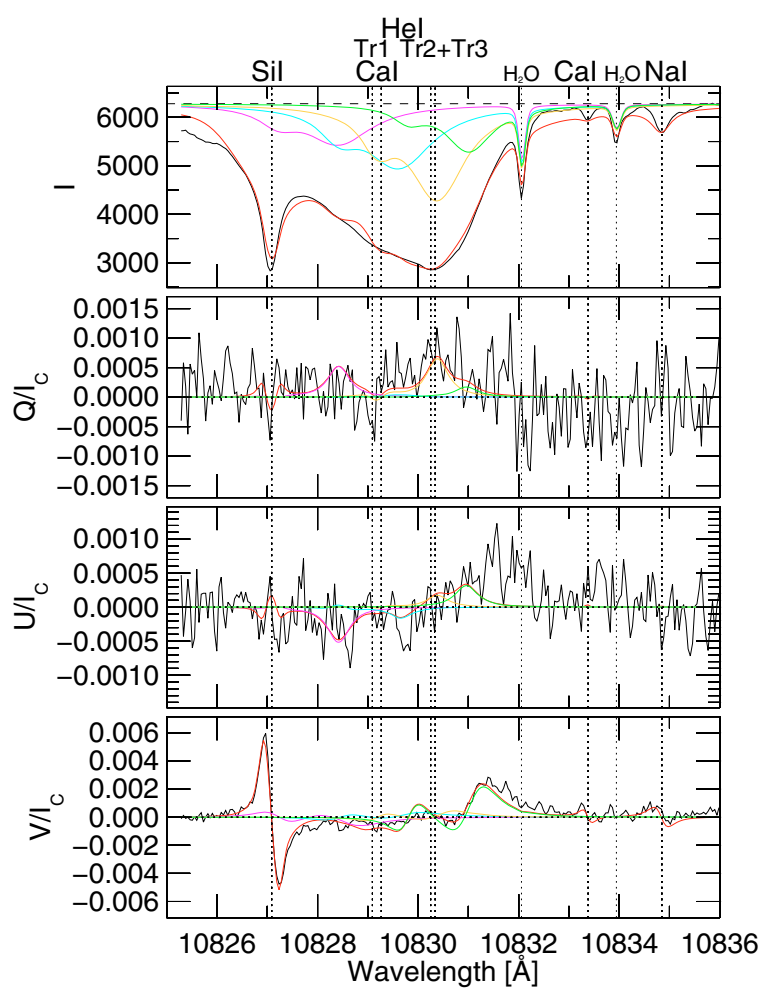

Fig. 11. Observed and inverted Stokes $I, Q, U$, and $V$ profiles at the location $\left(17^{\prime \prime}, 16^{\prime \prime}\right.$ of the map in Fig. 1. The solid black lines are the observed profiles, while the red lines are the fits involving four different atmospheric components to describe the He I lines (magenta, light blue, yellow, and green lines).

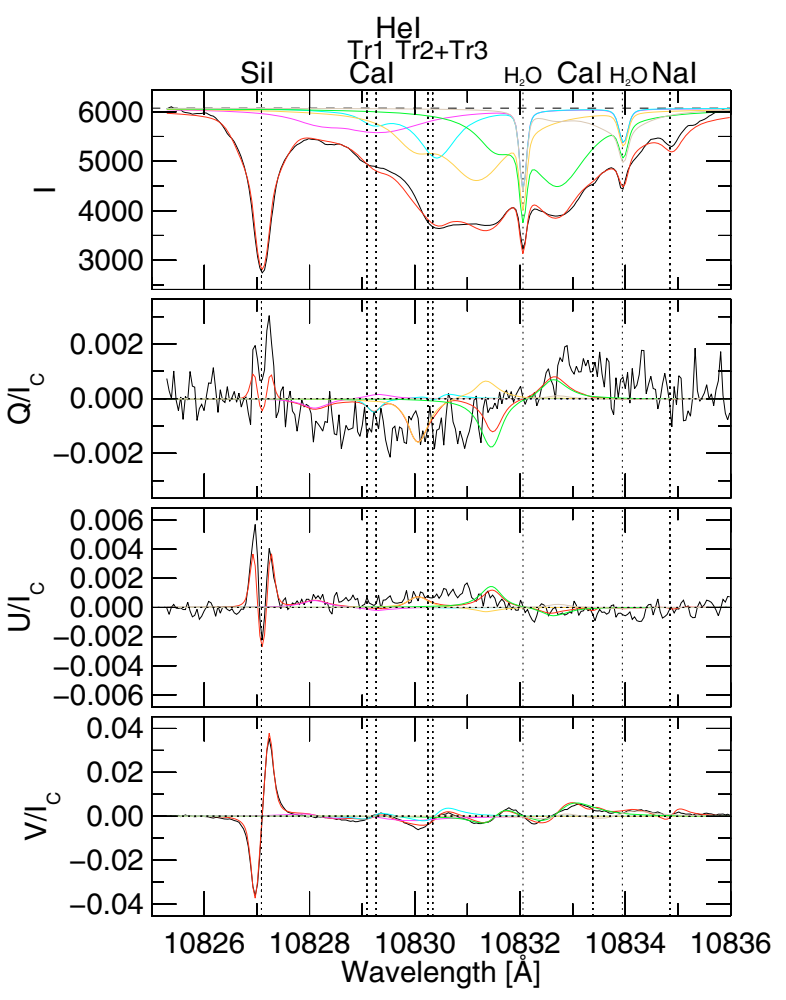

Fig. 12. Observed and inverted Stokes $I, Q, U$, and $V$ profiles at the location $\left(32^{\prime \prime}, 21^{\prime \prime}\right)$ of the map in Fig. 1. The solid black lines are the observed profiles, while the red lines are the fits involving five different atmospheric components to describe the He I lines (magenta, light blue, yellow, green, and dark gray lines).

region in the observed filament. The $v_{\mathrm{LOS}}$ is well retrieved for all profiles with a small error. The inclination angle $\gamma$ is retrieved with a somewhat larger error, but the inclinations of the different components of the magnetic field can often be distinguished. The error bars on the azimuth angle, $\chi$, are instead quite large, and it is often impossible to distinguish between the azimuths of the different magnetic components. This is mainly because of the complexity and the noise in the $Q$ and $U$ Stokes profiles. The information in the observations for retrieving the azimuth is limited. We therefore refrain from conclusions about the azimuthal direction of the magnetic field in the filament. There is also insufficient information in the profiles to distinguish between the field strength $B$ of the different components. Note that because Stokes $V$ is generally much stronger than $Q$ and $U, B \cos \gamma$ is probably the most reliably determined quantity, although we do not explicitly tabulate it.

The analysis of the observed polarization of the $\mathrm{He}$ I $10830 \AA$ multiplet in the filament, carried out by inverting the Stokes profiles, reveals different unresolved atmospheric components of the He lines, coexisting within each spatial resolution element $(\sim 1$ arcsec). The components are distinguished by their Doppler shifts, which generally differ by more than the sound speed. For more complex analyzed profiles we also tried to find a solution considering a broadened profile with some emission contributions distributed at the appropriate places, but the fits to the observed profiles were not as good as the ones we present in the paper. The main problem is reproducing the $I$ and $V$ profiles at the same time.

In this active filament we find profiles requiring up to five atmospheric components for a reasonable fit. Multiple unresolved magnetic components are found not only in filaments, but also 
Table 4. Mean values of the atmospheric parameters with their errors returned by the fits in Figs. 8-12.

\begin{tabular}{|c|c|c|c|c|c|c|c|c|c|}
\hline \multicolumn{4}{|c|}{ Fitness $f=2.20 \pm 0.05$} & \multicolumn{6}{|c|}{ Fitness $f=1.72 \pm 0.07$} \\
\hline \multicolumn{2}{|c|}{ Parameter } & \multicolumn{2}{|c|}{ Component 1} & \multicolumn{2}{|c|}{ Parameter } & \multicolumn{2}{|c|}{ Component 1} & \multicolumn{2}{|c|}{ Component 2} \\
\hline \multicolumn{2}{|c|}{$B(\mathrm{G})$} & \multicolumn{2}{|c|}{$164 \pm 88$} & \multicolumn{2}{|c|}{$B(\mathrm{G})$} & \multicolumn{2}{|c|}{$141 \pm 82^{a}$} & \multicolumn{2}{|c|}{$141 \pm 82^{\mathrm{a}}$} \\
\hline \multicolumn{2}{|c|}{$\gamma\left({ }^{\circ}\right)$} & \multicolumn{2}{|c|}{$121 \pm 20$} & \multicolumn{2}{|c|}{$\gamma\left({ }^{\circ}\right)$} & \multicolumn{2}{|c|}{$82 \pm 5$} & \multicolumn{2}{|c|}{$40 \pm 26$} \\
\hline & & \multicolumn{2}{|c|}{$11 \pm 51$} & \multicolumn{2}{|c|}{$x\left(^{\circ}\right)$} & \multicolumn{2}{|c|}{$36+5$} & \multicolumn{2}{|c|}{$38 \pm 40$} \\
\hline$v_{\text {LOS }}{ }^{x}$ & $\left.\mathrm{~m} \mathrm{~s}^{-1}\right)$ & \multicolumn{2}{|c|}{$3.0 \pm 0.1$} & \multicolumn{2}{|c|}{$v_{\mathrm{IOS}}\left(\mathrm{km} \mathrm{s}^{-1}\right)$} & \multicolumn{2}{|c|}{$-52+1$} & \multicolumn{2}{|c|}{$1.8 \pm 0.2$} \\
\hline & & \multicolumn{2}{|c|}{1.00} & \multicolumn{2}{|c|}{$\alpha$} & \multicolumn{2}{|c|}{$0.35 \pm 0.05$} & \multicolumn{2}{|c|}{$0.65 \pm 0.05$} \\
\hline & \multicolumn{9}{|c|}{ Fitness $f=2.35 \pm 0.06$} \\
\hline & Para & neter & Comp & onent 1 & Compc & nent 2 & Comp & onent 3 & \\
\hline & & G) & 135 & $45^{\mathrm{a}}$ & $135=$ & $45^{\mathrm{a}}$ & 135 & $\pm 45^{\mathrm{a}}$ & \\
\hline & & $\left({ }^{\circ}\right)$ & 83 & \pm 5 & 124 & +19 & & +19 & \\
\hline & & $\left.{ }^{\circ}\right)$ & -10 & \pm 2 & -16 & \pm 12 & & \pm 7 & \\
\hline & $v_{\mathrm{LOS}}(1$ & $\left.\mathrm{m} \mathrm{s}^{-1}\right)$ & -53 & \pm 1 & -30 & \pm 2 & 1.9 & \pm 0.2 & \\
\hline & & $x$ & $0.32=$ & $=0.04$ & $0.21=$ & 0.04 & 0.47 & +0.03 & \\
\hline & & & & ness $f=$ & $1.90 \pm 0$ & .08 & & & \\
\hline Para & neter & Comp & nent 1 & Comp & onent 2 & Comp & nent 3 & Comp & nent 4 \\
\hline & & 106 & $46^{a}$ & 106 & $\pm 46^{\mathrm{a}}$ & 106 & $46^{a}$ & 106 & $46^{a}$ \\
\hline & & & +7 & & \pm 5 & & \pm 5 & 160 & $=18$ \\
\hline & & -17 & \pm 30 & -17 & \pm 30 & & 19 & 35 & 11 \\
\hline$v_{\text {LOS }}{ }^{\lambda}$ & $\left.\mathrm{m} \mathrm{s}^{-1}\right)$ & -52 & \pm 2 & -18 & \pm 1 & & & & \\
\hline & & 0.17 & 0.04 & 0.23 & \pm 0.05 & 0.36 & 0.04 & 0.24 & 0.04 \\
\hline & & & & ness $f=$ & $1.79 \pm 0$ & & & & \\
\hline Parameter & Comp & nent 1 & Comp & nent 2 & Compc & nent 3 & Comp & onent 4 & Component 5 \\
\hline$B(\mathrm{G})$ & 244 & $=55^{a}$ & $244=$ & $=55^{a}$ & $244 \pm$ & $55^{a}$ & 244 & $\pm 55^{a}$ & $244 \pm 55^{a}$ \\
\hline$\gamma\left({ }^{\circ}\right)$ & $11=$ & $=11$ & 126 & \pm 10 & 89 & & 154 & \pm 17 & $154 \pm 18$ \\
\hline$x\left({ }^{\circ}\right)$ & $15=$ & $=47$ & $15=$ & $=40$ & $12 \pm$ & 40 & & \pm 9 & $3 \pm 65$ \\
\hline$v_{\mathrm{LOS}}\left(\mathrm{km} \mathrm{s}^{-1}\right)$ & -25 & \pm 1 & $3.6=$ & 0.5 & & & 67.3 & \pm 0.6 & $100 \pm 1$ \\
\hline$\alpha$ & $0.08=$ & $=0.01$ & $0.28=$ & 0.04 & $0.23=$ & 0.04 & 0.29 & \pm 0.03 & $0.11 \pm 0.03$ \\
\hline
\end{tabular}

Notes. ${ }^{(a)}$ The $B$ values in all components were forced to be equal (in each table).

above pores and elsewhere in active regions (Aznar Cuadrado et al. 2005; Lagg et al. 2007), but so far in other data sets never more than three components were needed. As Lagg et al. (2007) pointed out, the geometrical interpretation of these multiple downflow components is not clear-cut in the case of a line such as He I $10830 \AA$, which is generally optically thin. Thus the different components could be located at different horizontal positions within the resolution element, implying considerable fine structure in the filament. This fine structure in filaments at a subarc s scale has been reported earlier. See, e.g., Tandberg-Hanssen (1995) or Heinzel (2007), for reviews. These fine structures show a random motion with velocities of $5-10 \mathrm{~km} \mathrm{~s}^{-1}$, as deduced mainly from observations in the $\mathrm{H} \alpha$ line.

An alternative explanation is that the various components are located at different heights along the LOS. It may be argued that the He I line gets very strong in the filament and can hardly be considered to be optically thin, so that one should not be able to see through different layers so easily. We note, however, that the line becomes optically thick only at wavelengths at which the absorption is large, whereas it remains optically thin at neighboring wavelengths. Consequently, if the different layers absorb at wavelengths that are shifted by more than a Doppler width relative to each other, this explanation remains possible even if individual layers produce optically thick absorption.

Because in many cases the profiles have some optical thickness (they are formed over a range of heights), it is possible that gradients in velocity along the LOS can affect their shape. The influence of these gradients cannot be treated in the simple model employed here. A more general treatment with gradients might lead to good fits with a reduced number of components.

The inversions support the idea that the He components correspond to plasma trapped in different magnetic field lines, which may well be pointing in different directions along the LOS. Indeed, the coupling of the magnetic field vector between the individual components results in worse fits to the observed profiles, which supports this interpretation. Note though that some He profiles do not show a significant signal in Stokes $V$. They describe intensity features without a polarization counterpart.

Multiple magnetic components are fairly common in most parts of the observed filament and are often associated with strong blue- or redshifts corresponding to supersonic velocities. We measured downflow velocities of up to $100 \mathrm{~km} \mathrm{~s}^{-1}$ and upflows of up to $60 \mathrm{~km} \mathrm{~s}^{-1}$ along the line-of-sight. These supersonic up- and downflows always coexist with a He atmospheric component almost at rest $\left(-10<v_{\text {LOS }}<+10 \mathrm{~km} \mathrm{~s}^{-1}\right)$ within the same resolution element. Sometimes strong blue- and redshifted components are found to coexist in the same profile. Velocities of filament fine structures reported in the literature are low compared with these observed supersonic down- and upflows.

Strong motions and velocities in active region filaments were previously observed in the He I $10830 \AA$ lines. Observations of an erupting active region filament presented by Penn (2000) revealed the $\mathrm{He}$ I absorption line blue-shifted to velocities between 200 and $300 \mathrm{~km} \mathrm{~s}^{-1}$. In the same observation, the filament also showed internal motions with multiple Doppler components shifted by $\pm 25 \mathrm{~km} \mathrm{~s}^{-1}$. Redshifts of $30-60 \mathrm{~km} \mathrm{~s}^{-1}$ were reported by Penn \& Kuhn (1995) in an active filament during the decay phase of a flare in the He I 10830 absorption lines. We found the highest redshifts so far measured with the He I $10830 \AA$ line. In this respect, we emphasize the importance of the TIP-II instrument, which was used for the first time during this observation campaign. We were able to observe the full range of $\mathrm{He}$ absorption signatures and measure the highest downflow velocities 
thanks to its wider range of wavelengths compared to the original TIP instrument.

Acknowledgements. We thank Luca Teriaca for helpful discussions. We also thank Regina Aznar Cuadrado and Manolo Collados for their help during the observing run at the German Vacuum Tower Telescope at the Spanish observatory in Tenerife. C.S. thanks the IMPRS on Physical Processes in the Solar System and Beyond for the opportunity to carry out the research presented in this paper. This work was supported by the program "Acciones Integradas HispanoAlemanas" of the German Academic Exchange Service (DAAD project number D/04/39952), by the WCU grant No. R31-10016 from the Korean Ministry of Education, Science and Technology, and by the ASI/INAF contract I/05/07/0 for the program "Studi Esplorazione Sistema Solare".

\section{References}

Aznar Cuadrado, R., Solanki, S. K., \& Lagg, A. 2005, in Chromospheric and Coronal Magnetic Fields, ed. D. E. Innes, A. Lagg, S. K. Solanki, \& D. Danesy (ESA Publication Division), ESA SP-596, 49

Casini, R., López Ariste, A., Tomczyk S., \& Lites, B. W. 2003, ApJ, 598, 67

Charbonneau, P. 1995, ApJS, 101, 309

Collados, M., Lagg., A., Díaz García, J. J., et al. 2007, in The Physics of Chromospheric Plasmas, ed. P. Heinzel, I. Dorotovič, \& R. J. Rutten, ASP Conf. Ser., 368, 611

Heinzel, P. 2007, in The Physics of Chromospheric Plasmas, ed. P. Heinzel, I. Dorotovič, \& R. J. Rutten, ASP Conf. Ser., 368, 275
Kuckein, C., Centeno, R., Martínez Pillet, V., et al. 2009, A\&A, 501, 1113

Lagg, A., Woch, J., Krupp, N., \& Solanki, S. K. 2004, A\&A, 414, 1109

Lagg, A., Woch, J., Solanki, S. K., \& Krupp, N. 2007, A\&A, 462, 1147

Leroy, J. L. 1989, in Dynamics and Structure of Quiescent Solar Prominences, ed. E. R. Priest, (Dordrecht: Kluwer), 77

Lin, H., Penn, M. J., \& Kuhn, J. R. 1998, ApJ, 493, 978

López Ariste, A., \& Casini, R. 2006, in Solar Polarization 4, ed. R. Casini, \& B. W. Lites, ASP Conf. Ser., 358, 443

Low, B. C., \& Zhang, M. 2002, ApJ, 564, 53

Martínez Pillet, V., Collados, M., Sánchez Almeida, J., et al. 1999, in High Resolution Solar Physics: Theory, Observations, and Techniques, ed. T. R. Rimmele, K. S. Balasubramaniam, \& R. R. Radick, ASP Conf. Ser., 183, 264 Merenda, L., Trujillo Bueno, J., Landi DeglInnocenti, E., \& Collados, M. 2006, ApJ, 642, 554

Penn, M. J. 2000, Sol. Phys., 197, 313

Penn, M. J., \& Kuhn, J. R. 1995, ApJ, 441, 51

Rüedi, I., Solanki, S. K., \& Livingston, W. C. 1995, A\&A, 293, 252

Sasso, C., Lagg, A., \& Solanki, S. K. 2006, A\&A, 456, 367

Socas-Navarro, H., Trujillo Bueno, J., \& Landi Degl'Innocenti, E. 2004, ApJ, 612,1180

Solanki, S. K., Lagg, A., Woch, J., Krupp, N., \& Collados, M. 2003, Nature, 425 Tandberg-Hanssen, E. 1995, The Nature of Solar Prominences (Dordrecht: Kluwer Acad. Publ.)

Trujillo Bueno, J., Landi Degl'Innocenti, E., Collados, M., Merenda, L., \& Manso Sainz, R. 2002, Nature, 415, 403 